\title{
High-yield recombinant expression and purification of marginally soluble, short elastin-like polypeptides
}

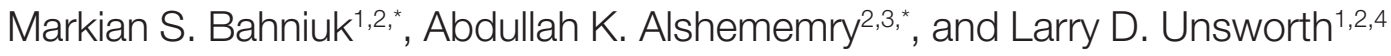 \\ ${ }^{1}$ Department of Biomedical Engineering, University of Alberta, Edmonton, Alberta, Canada, ${ }^{2}$ National \\ Institute for Nanotechnology, National Research Council, Edmonton, Alberta, Canada, ${ }^{3}$ Faculty of \\ Pharmacy and Pharmaceutical Sciences, University of Alberta, Edmonton, Alberta, Canada, and \\ ${ }^{4}$ Department of Chemical \& Materials Engineering, University of Alberta, Edmonton, Alberta, Canada
}

${ }^{*}$ M.S.B. and A.K.A. contributed equally to this work.

BioTechniques 61:297-304 (December 2016) doi 10.2144/000114482

Keywords: elastin-like polypeptides; recursive directional ligation; inverse temperature transitions; concatemerization; denaturing metal affinity chromatography; marginally soluble protein purification

Supplementary material for this article is available at www.BioTechniques.com/article/114482.

The protocol described here is designed as an extension of existing techniques for creating elastin-like polypeptides. It allows for the expression and purification of elastin-like polypeptide (ELP) constructs that are poorly expressed or have very low transition temperatures. DNA concatemerization has been modified to reduce issues caused by methylation sensitivity and inefficient cloning. Linearization of the modified expression vector has been altered to greatly increase cleavage efficiency. The purification regimen is based upon using denaturing metal affinity chromatography to fully solubilize and, if necessary, pre-concentrate the target peptide before purification by inverse temperature cycling (ITC). This protocol has been used to express multiple leucine-containing elastin-like polypeptides, with final yields of 250-660 mg per liter of cells, depending on the specific construct. This was considerably greater than previously reported yields for similar ELPs. Due to the relative hydrophobicity of the tested constructs, even compared with commonly employed ELPs, conventional methods would not have been able to be purify these peptides.

Elastin-like polypeptides (ELPS) are protein-based biopolymer analogs of mammalian elastin (1). The general architecture of an ELP sequence is any number of repeats of the amino acid sequence valine-proline-glycine-X-glycine (VPGXG), where $X$ can be any amino acid except proline (2). In elastin, this guest amino acid is typically valine. What makes this particular sequence of amino acids distinct is that it can reversibly undergo self-assembly in reaction to changes in local environmental factors such as temperature and salinity (1). The ELP amino acid chain typically exists in a random coil conformation in solution, but upon heating, it undergoes a conformational change, adopting a $\beta$-spiral conformation (3). These spirals then self-associate to form aggregates. Upon cooling, it is thought that ELPs revert to a random coil conformation and regain solubility.

The exact environmental conditions under which ELPs change their conformation and solubility depend greatly upon the number of repeats of the VPGXG sequence and the side chain chemistry of the amino acid in the guest amino acid position (2). This effectively means that ELPs can be customized to aggregate under very specific conditions and can be employed in a variety of situations. ELPs are being used in a number of applications, including protein purification, environmental sensing, customizable drug delivery vehicles, and as a method of targeting the delivery of drugs to specific areas (4-7). In addition to their flexibility, the preliminary biocompatibility of ELPs has been favorable and because they are made up of amino acids, there are few concerns about toxic degradation products (8). Another advantage of using protein-based polymers such as ELPs is that they can be produced recombinantly by inserting a DNA gene encoding an ELP into Escherichia coli, using the bacteria as

\section{METHOD SUMMARY}

This efficient and dependable method for the high-yield production and purification of short, marginally soluble, elastin-like polypeptides (ELPS) involves increasing the reliability of DNA concatemerization procedures and utilizing denaturing metal affinity chromatography to ensure these ELPs are fully soluble and recoverable from insoluble cell debris. If the ELPs are poorly expressed, this also serves as a pre-concentration step to maximize the ELP concentration before temperature cycling purification. 
molecular factories. Making these types of molecules using recombinant methods allows for an unprecedented level of control over the final product compared with traditional polymer synthesis and can allow for the straightforward incorporation of various biofunctional moieties, including targeting, cell penetrating, and enzymatically cleavable sequences.

The purification of recombinantly produced proteins is typically the largest bottleneck in their production, but with ELPs, their reversible solubility can be exploited to partially simplify purification under the correct conditions. This approach is known as inverse temperature cycling (ITC) and was first demonstrated by Meyer and Chilkoti (9). While ELPs and their potential applications are attractive and worth pursuing, there has until now been no published systematic protocol for expressing and purifying marginally soluble, short ELPS, and in our experience, standard protocols are inadequate for this subset of ELPs.

DNA sequences encoding for ELPs are by their very nature highly repetitive and are often rich with $\mathrm{G}-\mathrm{C}$ base pairs. These two characteristics makes it difficult to work with these types of sequences in the lab and also prohibitively expensive to synthesize large ELP genes commercially. Techniques such as PCR, which depends on the melting and re-annealing of doublestranded DNA, may not function well, if at all, for ELP-encoding DNA sequences. Fortunately, through the clever application of specific restriction enzymes, short commercially sourced ELP genes can be concatemerized together. This process is known as recursive directional ligation (RDL) (10). Here, we describe a protocol featuring modifications to RDL designed to address technical bottlenecks experienced in our lab, including inefficient restriction enzyme cleavage due to DNA methylation, significant numbers of incorrect vector-only clones during attempted ELP sequence concatemerization, and minimal Sfil cleavage when using only one cleavage site in the expression vector modifying sequence. Our modifications greatly increase the overall efficiency of the RDL process while minimizing time- and reagent-intensive steps, such as modifiedexpression-vector linearization and mass colony screening.

The protein sequences created from these difficult-to-process DNA sequences can also be troublesome to deal with. Given that ELPs are generally hydrophobic regardless of their conformation, even routine lab procedures such as spin concentration, dialysis, and long-term storage become more complicated. Care must also be taken to not lose ELPs due to non-specific adsorption to sample tubes during routine handling. The ELPs covered here are highly repetitive, typically uncharged, relatively hydrophobic, and can spontaneously precipitate out of solution when stored at high concentrations at temperatures as low as $4^{\circ} \mathrm{C}$ for long periods of time. While these characteristics are integral to the unique reversible solubilization of ELPs, they also make their purification and downstream sample handling a delicate affair.

When ELPS have been purified successfully, for the most part the guest amino acids in these constructs fall toward the middle of Urry's ELP hydrophobicity scale (11). While ITC purification has been sufficient for these types of constructs, it is not applicable to all ELPs. Successfully employing an ITC-only purification procedure means the ELP construct and its expression have to meet certain criteria: The ELP must not end up in the insoluble fraction of the cell lysate during the lysis procedure; the ELP must be in its soluble monomer form in the cell lysate; the phase transition needs to be triggered in the cell lysate under reasonable temperature and salinity conditions; and the protein must be expressed at a concentration high enough for a phase transition to be possible under reasonable conditions but must also be low enough to avoid significant depression of the transition temperature as well as the formation of inclusion bodies. Not all ELP purifications meet these criteria. If an ELP is poorly expressed, or if it contains guest amino acids that are significantly more hydrophilic or hydrophobic than those commonly employed in the literature, or if the ELPs are significantly longer or shorter than what is commonly used, the transition temperature of the ELP construct may be too high or too low for an ITC-only approach.

Some work has been carried out on shorter, hydrophobic ELPs using maltose binding protein (MBP) as a solubility and affinity-purification partner as a way to circumvent the above issues (12). This has, however, introduced another complication in that the MBP must then be separated from the hydrophobic ELP, an approach with varying levels of success depending on the ELP construct in question (12). More recently an ITC-only purification approach has been used to produce the ELPs without the interfering MBP, but the yields of purified protein severely limit the applications of such an approach (13).

\section{Materials and methods}

A complete listing of the materials used in this study and a detailed protocol are provided in the Supplementary Material.

\section{ELP gene concatemerization} and cloning

A synthetic oligonucleotide encoding for 10 repeats of VPGLG $\left(L_{10}\right)$ was purchased from Integrated DNA Technologies (Coralville, IA). Due to its length the oligonucleotide was provided in a pIDT-blue plasmid. This plasmid was transformed into XL10 Gold competent E. coli cells (Agilent Technologies, Santa Clara, CA), and the cells were grown in liquid culture to produce large amounts of the ELP-containing plasmid. The ELP gene was obtained from the plasmid by double digesting it with the EcoRI and HinDIII restriction enzymes (all restriction enzymes were purchased from New England BioLabs, Ipswich, MA) and purified by agarose gel electrophoresis. The gene was ligated into a pUC19 vector (Bio Basic, Ontario, Canada) that was also digested by the same restriction enzymes. Correct gene insertion was confirmed by double digestion with restriction enzymes Bgll and Ndel.

Oligomerization of the ELP gene was carried out using a modified recursive directional ligation (RDL) scheme. Vector DNA was linearized using PfIMI, purified using gel electrophoresis, digested again with PfIMl, and dephosphorylated using Antarctic phosphatase (New England Biolabs). Insert DNA was double-digested with PfIMl and Bg/l and purified using gel electrophoresis. The two ELP-containing genes were ligated together and transformed into XL10 Gold cells. A double digest using PfIMl and Bg/l confirmed successful gene concatemerization. This $\mathrm{RDL}$ process was repeated to generate ELP genes with increasing numbers of repeats.

For the RDL-generated ELP genes to be inserted into a pET-25b(+) expression vector (EMD Millipore, Ontario, Canada), a 
short modifying sequence containing two Sfil cleavage sites along with the $\mathrm{N}$ - and $\mathrm{C}$-terminal sequences of the ELP was first added. As with the $L_{10}$ gene above, the synthetic oligonucleotide was purchased commercially (Integrated DNA Technologies), supplied in a vector, transformed into XL10 E. coli, and the sequence of interest was isolated using the EcoRI and Ndel restriction enzymes. The expression vector was similarly linearized and then ligated with the isolated modifying sequence. A post-ligation digest with BamHI before transformation was used to greatly reduce the number of vector-only clones. Vector modification was confirmed by single digests with either BamHI or Sfil.

To prepare for insertion of the ELP gene into the modified expression vector, the vector was digested with Sfil, purified by gel electrophoresis, digested again, and then dephosphorylated. The insert was prepared in the same manner as the inserts for RDL. The two were ligated together, and a post-ligation Sfil digest was performed before transformation to reduce the number of incorrect colonies observed after cloning. Restriction enzyme digestions and DNA sequencing confirmed the final plasmid sequences.

\section{Expression and purification}

ELP expression vectors were transformed into OneTouch BL21 (DE3) E. coli (Invitrogen, Carlsbad, CA) cells, and 1 $L$ cultures of these cells were grown in Terrific Broth (TB) (Thermo Fisher Scientific, Waltham, MA) supplemented with 100 $\mu \mathrm{g} / \mathrm{mL}$ ampicillin (Thermo Fisher Scientific) and $10 \mathrm{mM}$ L-proline (Sigma-Aldrich, St. Louis, MO). Expression was induced for 24 h using $2 \mathrm{mM}$ isopropyl $\beta$-D-1-thiogalactopyranoside (Thermo Fisher Scientific).

Purification was achieved by first performing denaturing metal-affinity chromatography and eluting the ELPs using an imidazole step gradient. Buffered $8 \mathrm{M}$ urea (Thermo Fisher Scientific) was used to lyse the cells and ensure the ELPS were fully soluble and free from any inclusion bodies. The urea was removed during the extensive column washing. Eluents were screened for the presence of ELPs by gel electrophoresis or by heating them to room temperature or $37^{\circ} \mathrm{C}$, depending on the construct, and observing which samples turned reversibly cloudy. Samples confirmed to contain ELPs were combined and then subjected to one round of ITC for final purification. The temperature used to cause ELP aggregation varied depending on the construct. The purification was confirmed with denaturing PAGE (SDS-PAGE) and the ELP concentrations were measured by sample absorbance at $280 \mathrm{~nm}$.

\section{Results and discussion}

Many of the design considerations for new ELP sequences have been discussed in detail previously (10). Briefly, the design of the initial DNA sequences should take into account $E$. coli codon bias; that is, the frequency of the employed triplet codons should reflect the naturally occurring frequency of their corresponding tRNAs. The ends of the ELP DNA sequences should be designed such that oligomerization of the DNA eliminates the PfIMI and $B g / l$ cleavage sites without introducing another cut site for a restriction enzyme employed downstream. While these restriction enzymes are used here to facilitate ELP gene oligomerization, they are not the only two that could be used. In addition to the published guidelines, if individual oligonucleotides are being used, rather than genes already contained in plasmids, the oligonucleotides should be ordered in a semi-purified state. If the oligonucleotides are ordered with only standard desalting as the purification method, there is a high probability that an undesirable heterogeneous product will be obtained because of the highly repeated sequence.

The basic workflow of RDL has been based on the work of Meyer and Chilkoti (10). Modifications have been introduced to both maximize restriction enzyme digest efficiency by minimizing interference by methylation and significantly decrease the likelihood of incorrect background colonies by maximizing vector linearization and purity. This drastically reduces the number of colonies that must be screened in order to find the correct RDL product. We found that methylation was less of a concern when using XL10-Gold E. coli as opposed to the XL1-Blue strain. Before switching host cell lines, restriction enzyme digests were usually only 30\%-40\% efficient. This drastically reduced the yield of linearized vector and correctly digested inserts necessary for RDL. The number of background colonies was decreased by gel purifying the digested vectors, repeating the linearization procedure, and then dephosphorylating the vector. These

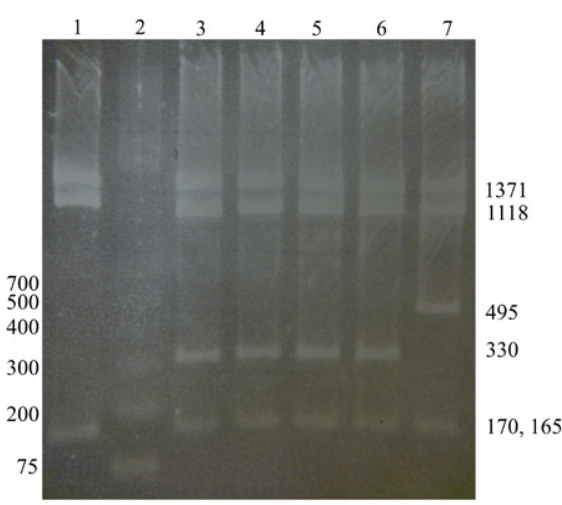

Figure 1. Sample results demonstrating a successful doubling of an elastin-like polypeptide (ELP) $\mathrm{L}_{10}$ insert to $\mathrm{L}_{20}$. Plasmids were digested with PfIMI and $B g / l$ for $1 \mathrm{~h}$ and then run on a $2 \%$ agarose gel before being stained with ethidium bromide. This digest should yield 170, 1118 and $1371 \mathrm{bp}$ bands from the vector, plus an ELP band whose size will vary. In this instance, the $L_{10}$ gene was 165 bp long, the $L_{20}$ gene was 330 bp, and the $L_{30}$ gene was $495 \mathrm{bp}$. Lane 1 is a positive control digest of pUC19- $L_{10}$, Lane 2 is the molecular weight ladder, and Lanes 3-7 are clones from the RDL reaction concatemerizing $L_{10}$ to $L_{20}$ Based on the increase in band sizes, Lanes 3-6 contain the genes for $L_{20}$, while Lane 7 likely contains an $L_{30}$ gene. Note that the $170-b p$ vectorderived fragment is clearly visible in Lanes 3-7 but overlaps the $L_{10}$ gene in Lane 1 . The $L_{30}$ is the result of a double insertion of the ELP $L_{10}$ gene into a single cloning vector. This is not an uncommon occurrence while performing RDL using the recommended molar ratios of insert to vector and may actually be beneficial in some cases.

additional steps help to reduce the number of background colonies resulting from both uncut vector DNA and re-closed empty vectors. Without this additional effort, the number of clones containing only the vector completely eclipsed the number of correctly ligated clones. These precautions reduced the proportion of incorrect clones from $\sim 95 \%$ to $<5 \%$. Figure 1 shows the results of an RDL colony screening procedure and clearly demonstrates the complete lack of empty vector clones. The time required to screen clones to find a correct ligation is greatly reduced by performing all of the steps above to reduce both uncut vector and religated DNA. Useful controls for the RDL cloning procedure consisting of DNA ligation reactions where the ELP insert and/or ligase enzyme are replaced by water can be used to assess the amount of empty vector that makes it through the ligation procedure. The sequence of the $L_{10}$ gene used as an example in the detailed protocol is shown in Supplementary Figure S1.

Modification of the expression vector before insertion of the ELP gene is a 
necessary step of RDL (10). In order for the RDL strategy to generate complete repeats of an ELP gene, some of the DNA encoding for $\mathrm{N}$ - and $\mathrm{C}$-terminal amino acids cannot be included in the original ELP oligonucleotide and must be added in the expression vector. This also allows for the introduction of various $\mathrm{N}$ - and $\mathrm{C}$-terminal modifications without needing to redo the entire RDL procedure. Two Sfil sites and a spacer of appropriate length need to be added to the pET-25b(+) vector so that the RDL-derived ELP gene can be inserted into the expression vector. This is a subtle but very significant departure from previous RDL methods (10). The Sfil restriction enzyme is quite uncommon in its structure, and the majority of the literature on this enzyme states that efficient cleavage of DNA by Sfil only occurs when 2 of its recognition sites are present and separated by roughly 200-300 bp (14-17). The Sfil enzyme works by simultaneously cleaving at both recognition sites, which results in one compatible set of sticky ends in the vector. Some RDL literature suggests that a single Sfil recognition site is sufficient $(9,10)$; however, efficient and reproducible cleavage was only observed in our work when two appropriately spaced recognition sites were located in the same stretch of DNA. A vector with a single Sfil site was only cleaved with 10\% efficiency, whereas a vector with 2 Sfil sites and a spacer had a cleavage efficiency of $\sim 100 \%$. Using a vector with a single Sfil site and doping the digest reaction with a short double-stranded DNA oligonucleotide also containing an Sfil cut site did not significantly improve vector linearization in our experience. The modification sequence we designed (detailed in Supplementary Figure S2) contained the DNA for the Nand C-terminal amino acids of the ELP gene, the two Sfil restriction sites and their spacer, a tobacco etch virus protease recognition site, and a polyhistidine tag.

ELPs were expressed by a conventional IPTG induction method. There are other protocols that suggest uninduced expression yields more ELP (18); however, we have found that IPTG induction of these constructs and a $24 \mathrm{~h}$ expression period dramatically increased expression yields. Numerous parameters were tested, including expression time, $\mathrm{OD}_{600}$ at time of induction, incubation temperature, IPTG concentration, and uninduced expression. The expression conditions described here represent those that worked well for the corresponding constructs; however, they may not be ideal for the expression of other ELPs, and some sequence specific optimization may be required.

In most circumstances, standard Trisglycine SDS-PAGE was sufficient unless small ELPs such as $L_{20}$ were being produced. In this case, Tris-tricine electrophoresis allowed for superior resolution of the low molecular weight products. The tricine SDS-PAGE method has been modified in order to scale down the gel sizes for a Bio-Rad Mini-Protein Tetra Cell system (see protocol in Supplementary Material) (19). In all cases, gels were negatively stained with $\mathrm{CuCl}_{2}$ (20) as some literature suggests conventional Coomassie staining does not work on ELPs (21).

Denaturing nickel affinity chromatography was the first method used to purify the ELPS. It was chosen because it can act as a pre-concentration step for poorly expressed ELPs and also because the use of urea helps to ensure that individual ELPs, as well as any inclusion bodies, are fully solubilized. Especially with very hydrophobic ELPs, there is a chance their transition temperature is below room temperature and that they would end up

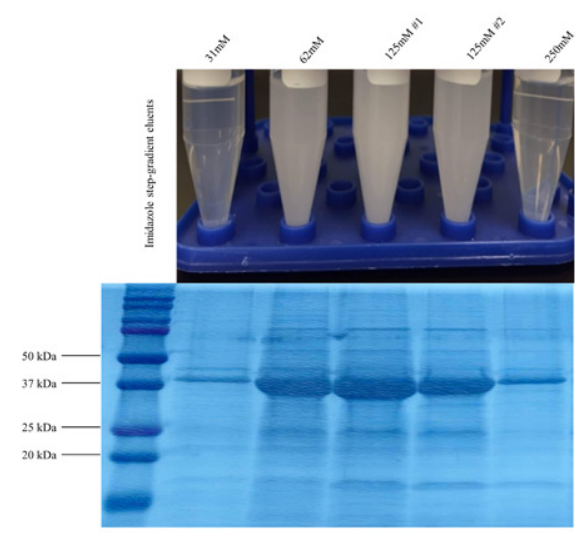

Figure 2. Demonstration of the concentration effects for elastin-like polypeptide (ELP) $\mathrm{L}_{80}$ containing metal-affinity eluents. The sample tubes represent various imidazole step-gradient eluents from a purification of ELP $L_{80}$ from the Ni-NTA column left at room temperature for a few minutes. From left to right, the tubes and their corresponding lanes on a $\mathrm{CuCl}_{2}$-stained $12 \%$ SDS-PAGE gel are the $31 \mathrm{mM}, 62 \mathrm{mM}$, $125 \mathrm{mM} \# 1,125 \mathrm{mM} \# 2$, and $250 \mathrm{mM}$ imidazole eluents in $25 \mathrm{mM}$ Tris, $50 \mathrm{mM} \mathrm{NaCl}$, pH 8.0. This ELP has a molecular weight of $39.7 \mathrm{kDa}$; $125 \mathrm{mM} \# 1$ and $125 \mathrm{mM} \# 2$ refer to sequential fractions of the same eluent. Sample volumes of $15 \mu \mathrm{L}$ were loaded onto the SDS-PAGE gel. The cloudiness was easily reversed simply by placing the tubes at $4^{\circ} \mathrm{C}$ for a few minutes. being inadvertently removed from solution when centrifuging away insoluble cellular contaminants. If the ELPs are poorly expressed, their low concentration could potentially cause the mass aggregation temperature to be prohibitively high, thus making an exclusively ITC-based purification impossible. We found that an overnight binding of the crude cell lysate to the nickel affinity column beads is crucial to recovering large amounts of ELP. In other ELP purification protocols polyethyleneimine is used to remove DNA; however, it is not necessary for denaturing nickel affinity chromatography, and in our experience, polyethyleneimine actually precipitated the hydrophobic ELPs as well as the DNA. Other ELP constructs may elute at different imidazole concentrations, so gradient elutions may need to be optimized for ELP constructs not used in this study. Figure 2 illustrates the elution of a leucine-containing ELP and the reversible cloudiness that corresponds to eluents containing significant amounts of the protein.

ITC was then used to purify the ELPS from any contaminating proteins left over from the metal affinity chromatography. This is a common method for purifying ELPS, and it is readily employed throughout the literature. ITC will only work, however, if the ELPs are sufficiently concentrated so that they can undergo a phase transition under reasonable conditions and they are not so hydrophobic as to have a mass aggregation temperature below the freezing point of water. For our hydrophobic proteins, the transition temperatures can fall below room temperature and as such, supplementary $\mathrm{NaCl}$ and heated centrifugation were not always necessary. Because of the immobilized metal affinity chromatography, only one round of ITC was necessary to fully purify the ELPS. Figure 3 shows the affinity chromatography eluents and their subsequent purification using only one round of ITC. Determining the appropriate conditions for purifying an ELP using ITC may require some optimization. Mass spectrometry was carried out on the purified ELPs, and we found that most leucine-containing ELPs did not elicit a strong signal. $\mathrm{L}_{40}$, however, did respond well, and its measured molecular weight was 21,155 Da, which matches with the expected molecular weight of 21,125 , within the margin-of-error of the spectrometer. Because all of the leucine- 


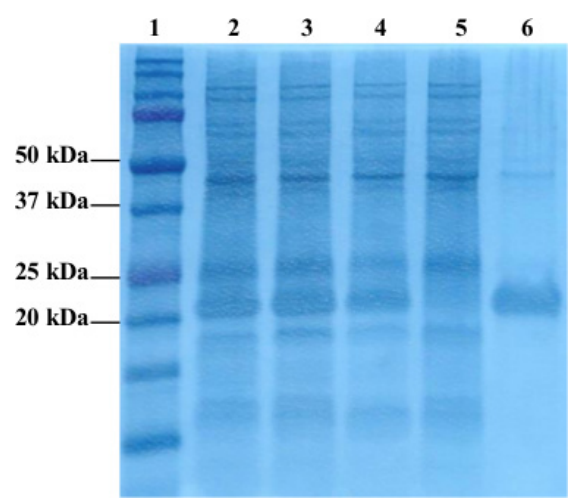

Figure 3. Polyacrylamide gel demonstrating the results of metal-affinity and inverse temperature cycling (ITC) purification of elastin-like polypeptide (ELP) $\mathrm{L}_{40}$. Lane 1 was loaded with $5 \mu \mathrm{L}$ of the molecular weight ladder. Lanes $2-4$ were loaded with $15 \mu \mathrm{L}$ samples of eluents from the metal-affinity purification eluted with 62 $\mathrm{mM}$ imdiazole (Lane 2) and $125 \mathrm{mM}$ imidazole (Lanes 3 and 4) and showing the ELP band at $21 \mathrm{kDa}$. Lane 5 shows the impurities from $15 \mu \mathrm{L}$ eluent collected after the hot spin in the ITC procedure, and Lane 6 was loaded with $15 \mu \mathrm{L}$ of the final purified ELP sample after the cold spin and shows the ELP primarily as a monomer but with a small amount of dimer as well. Samples were run on a Tris-tricine gel and stained with $\mathrm{CuCl}_{2}$.

containing ELPs were derived from the same DNA sequence, this result suggests there are no reasons to doubt the identity of the other ELPS.

Existing ITC purification approaches depend on triggering an ELP phase transition in the E. coli cellular lysate. This requires the solubility, transition temperature, and concentration of the ELP to fall within a certain range of values for a successful transition to be possible. For ELPS with a small number of repeats, and thus a high transition temperature, this approach may not be feasible. Additionally, constructs with strongly hydrophobic guest amino acids may have transition temperatures near or below the freezing point of water and, as such, the ITC-only approach may not be viable. In this case, the ELP may be inadvertently removed from the cell lysate when it undergoes centrifugation to remove the insoluble cell debris from the soluble fraction. The result could be the same if the ELPs are overexpressed and end up in inclusion bodies. Preliminary purifications of the leucine-containing ELPs using conventional non-denaturing tip sonication and ITC-only purifications yielded minimal amounts of protein. As such, a new purification approach was necessary.

The advantage of our method is that it is applicable to a wider range of ELP constructs than ITC-only purification procedures. The protocol uses denaturing metal affinity chromatography as the first step in purification. This ensures the ELPs are fully soluble upon cell lysis, in case they have very low transition temperatures or are expressed as inclusion bodies. Should there be an additional, chaotrope-sensitive domain appended to the ELP, non-denaturing metal affinity chromatography may be an acceptable substitute. The chromatography can also be used to concentrate the ELPS in order to lower their transition temperature to more reasonable levels should they be poorly expressed or have high transition temperatures at moderate concentrations. For instance, this hybrid method was used to successfully purify a conventional hydrophilic ELP construct in our lab that was so poorly expressed ( $0.35 \mathrm{mg}$ per liter of cells) that standard ITC-only purification failed because the phase transition could not be triggered due to such a low ELP concentration. The chromatography was key to successful purification because it allowed us to significantly pre-concentrate the ELP before attempting ITC. Our hybrid purification approach has been used to successfully purify as much as $660 \mathrm{mg}$ of marginally soluble, short ELPs per liter of cells. While this value varied depending on the specific length of the construct, observed yields ranged $250-660 \mathrm{mg} / \mathrm{L}$. These results are a significant improvement over our in-house attempts to apply conventional ITC-only purification methods to this class of ELPs as well as previously reported yields of comparable marginally soluble, short ELPs that produced roughly 10 and $35 \mathrm{mg}$ per liter of cells, respectively $(12,13)$. The first purification step serves to remove a large number of contaminating proteins that would otherwise be carried over into the subsequent ITC step. These contaminating proteins typically would require multiple rounds of ITC to be removed, but by employing affinity chromatography first, a pure product can be obtained after one round of ITC. Furthermore, by using a small polyhistidine tag for the affinity purification, rather than a larger multi-kilodalton partner such as MBP or glutathione S-transferase, it may not be necessary to remove the affinity tag. This reduces the amount of sample preparation and eliminates potential complications if the ELP complexes with its purification partner after the two are cleaved from one another.

Our new protocol is designed to improve the overall efficiency of the RDL DNA concatemerization process by using a combination of denaturing metal affinity chromatography and ITC to purify ELPs that would otherwise be unpurifiable using an ITC-only procedure. It does not require the use of large solubility or affinity partners and only requires a short polyhistidine tag, which may not necessarily need to be removed depending on the downstream applications for which the ELPs will be used. This protocol is designed specifically to expand upon ITC-based purifications to allow for the rapid purification of constructs that are: (i) poorly-expressed; (ii) expressed in inclusion bodies or as ELP aggregates; (iii) short constructs with high transition temperatures; and/or (iv) ELPs with strongly hydrophobic guest amino acids.

\section{Author contributions}

M.S.B. and L.D.U contributed to the conception of the study. M.S.B. and A.K.A. contributed to the development, analysis, and execution of the experiments. M.S.B., A.K.A., and L.D.U. wrote and edited the manuscript.

\section{Acknowledgments}

The authors would like to acknowledge Wayne Materi, Valentyna Semenchenko, Angela Marilyn Brigley, and Catherine de Guzman for their advice and technical assistance. This work was supported by the Natural Sciences and Engineering Research Council of Canada Discovery grant \#RES0011279. M.S.B. was supported by the Alberta Innovates Technology Futures graduate student scholarship in nanotechnology \#200801440. A.K.A. was the recipient of the Ministry of Higher Education and King Saud University Scholarship, Saudi Arabia.

\section{Competing interests}

The authors declare no competing interests.

\section{References}

1. Urry, D.W. 1988. Entropic elastic processes in protein mechanisms. I. Elastic structure due to an inverse temperature transition and elasticity due to internal chain dynamics. J. Protein Chem. 7:1-34

2. Urry, D.W., C.H. Luan, T.M. Parker, D.C. Gowda, K.U. Prasad, M.C. Reid, and A. Safavy. 1991. Temperature of polypeptide inverse temperature transition depends on mean residue hydrophobicity. J. Am. Chem. Soc. 113:4346-4348.

3. Urry, D.W., T.L. Trapane, and K.U. Prasad. 1985. Phase-structure transitions of the elastin polypentapeptide-water system within 
